\title{
Metal artifacts reduction in X-ray CT based on segmentation and forward-projection
}

\author{
Shoukat Nawaz, Jian Fu* and Dekai Fan \\ Research Center of Digital Radiation Imaging and Biomedical Imaging, Beijing University of \\ Aeronautics and Astronautics, 100191 Beijing, People's Republic of China
}

\begin{abstract}
X-ray computed tomography (CT) is a powerful clinical diagnosis tool and has been used widely in many clinical and biological settings. Metal artifacts, caused by high density implants, are commonly encountered in clinical CT applications, thereby affecting the detection of abnormal structures and undermining CT's diagnostic value. In this paper, we developed a metal artifact reduction approach based on image segmentation and forward-projection. We further demonstrate the usefulness of our approach by using a biomedical specimen consisting of muscles, bones and metals. Our aim is to remove the inaccurate metal artifact pixels in the original CT slices and exactly reconstruct the soft-tissue using the forward projections with no metal information. During the reconstruction, artifacts are reduced by replacing the metal projection using the forward projection. The presented work is of interest for CT biomedical applications.
\end{abstract}

Keywords: Computed tomography, metal artifacts, image segmentation, forward-projection

\section{Introduction}

X-ray computed tomography $(\mathrm{CT})$ has been used widely in diagnostic radiology since the invention by Hounsfield in 1972 [1-26]. Because of its excellent performance on calculation speed and image quality, filtered back-projection (FBP) [2] has been the most popular reconstruction algorithm employed in CT. Theoretically, exact reconstruction can be obtained by the FBP algorithms if the ideal projection data is acquired by the CT hardware. However, it is not possible in practice for various reasons. First, the projection data is measured by a finite number of detector channels using finite beam-width over a finite rotation interval. Second, an x-ray tube source emits a continuous spectrum resulting in a phenomenon called beam hardening. Third, the measurements are noisy and involve scattered radiation. All these factors contribute to CT imaging artifacts [10-13]. Fortunately artifacts due to these effects are usually small, making FBP reconstructions are quite reasonable to accept.

However, the FBP reconstruction of highly-attenuated objects such as metal implants in soft-tissues often contains streaking and star-shaped artifacts $[5,14,22]$. These artifacts tend to reduce image quality and obscure valuable details. Therefore, effective metal artifacts reduction strategies are needed.

* Corresponding author: Jian Fu, Research Center of Digital Radiation Imaging and Biomedical Imaging, Beijing University of Aeronautics and Astronautics, Beijing 100191, People's Republic of China. Tel.:86-10-924339095; Fax: 86-10-82339095; E-mail: fujian706@buaa.edu.cn.

0959-2989/14/\$27.50 @ 2014 - IOS Press and the authors. 
During the last three decades, several approaches have been proposed to eliminate or reduce undesirable effects by metallic objects on CT images. These approached are referred to as metal artifact reduction (MAR) techniques. They can be classified into two major categories: iterative methods $[3,4,7,8]$ and the projection compensation methods $[6,9,16]$. The latter generates metal-free projection data and then reconstruct a metal-removed CT image based on the FBP algorithm. However, this approach may distort the resultant CT image and introduce new artifacts. On the other hand, iterative approaches can achieve better image quality than the projection methods. However, they are based on some strict assumptions and will become invalid once the assumptions are not met.

In this paper, a metal artifact reduction approach based on image segmentation and forward-projection is proposed. The usefulness of this approach is demonstrated by a CT imaging experiment using a biomedical specimen consisting of muscles, bones and metals. The presented work is of interest for CT biomedical applications. This paper is organized as follows. In Section 2, the proposed approach is described. In section 3, experimental results are presented and analyzed. Finally, conclusion and discussion are presented in Section 4.

\section{Methods and materials}

The correction principle and the working procedure of the proposed MAR approach are depicted in Figure 1. The basic idea is to remove the inaccurate metal artifact pixels in the original CT slices and exactly reconstruct the soft-tissue using the forward projections with no metal information.

It consists of six steps, as described below.

\subsection{Initial image reconstruction}

A two-dimensional cross-section of three-dimensional object can be described by a complex refractive index distribution $n(x, y)=1-\delta(x, y)+i \beta(x, y)$, where $x$ and $y$ describe the coordinate system of the sample. In x-ray CT, one measures the line integral of the imaginary part $\beta$ by recording the intensity image using $\mathrm{x}$-ray detector. These line integral measurements are also called projection images. Reconstruction algorithm aims to retrieve $\beta$ from the measured projection images.

When the raw projection data with metal information are available after CT scanning, the initial CT image can be reconstructed by the following FBP formula:

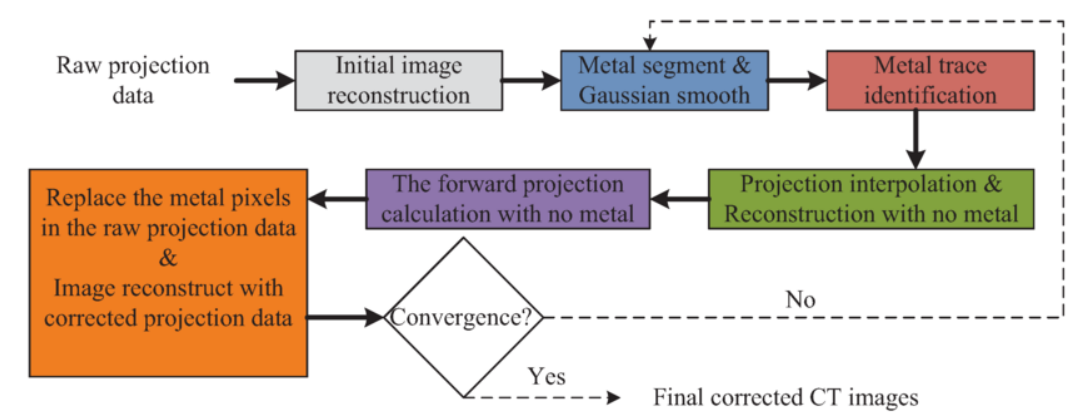

Fig. 1. The correction principle and the working procedure of the proposed metal artifacts reduction approach. 


$$
\beta_{i}(x, y)=\frac{1}{U^{2}} \int_{0}^{2 \pi} F T^{-1}(F T(P(\theta, s)) \times F T(h(s))) d \theta .
$$

Where $U$ is the geometrical factor determined by the scanning configuration of the CT systems. FT and $F T^{-1}$ are the Fourier transform pair. $P(\theta, s)$ represents the line integral measured by the detector channel $s$ at angular position $\theta . h$ is the ramp filter.

Figure 2 presents an example of initial CT reconstruction with metal artifacts, which is described in detail in the "Experiment" section. It is apparent that some structural details are difficult to be recognized due to the presence of metal artifacts.

\subsection{Metal segmentation from initial CT image}

As shown in Figure 2, the grey values corresponding to the metal pixels in the initial CT image are much higher than those of soft tissues. Hence, by threshold technique, the initial image $\beta(x, y)$ can be segmented and a characteristic image $\beta_{m}(x, y)$ is obtained and defined as:

$$
\beta_{m}(x, y)= \begin{cases}1 & \text { if } \beta_{i}(x, y)>\text { threshold } \\ 0 & \text { otherwise }\end{cases}
$$

Figure 3(a) demonstrates the metals segmented from the initial CT image. After segmentation, softtissue and bones disappear from the image. In order to avoid the effect of noise on segmentation, a Gaussian filter may be used to smooth the image before this operation. In addition, morphological image processing operators, such as erosion or dilation, may be applied to the set of metal pixels to increase the accuracy of the segmentation.

\subsection{Metal trace identification}

Based on the segmented metals $\beta_{m}(x, y)$ in Figure 3(a), the metal-trace in the projection space $(\theta, s)$ can be identified by the reprojection method [2] and expressed by $s_{m}(\theta)$, depicted in Figure 3(b).

$$
s_{m}(\theta)= \begin{cases}1 & \text { for metal-trace } \\ 0 & \text { otherwise }\end{cases}
$$

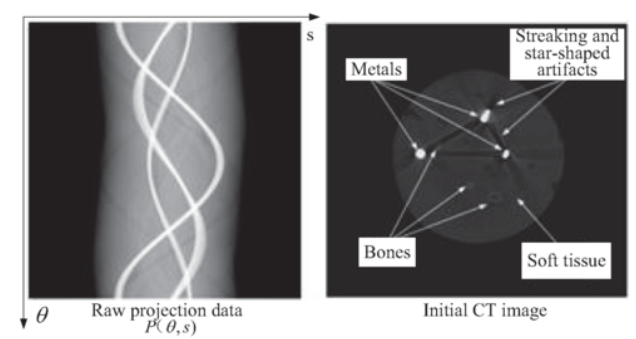

Fig. 2. The raw projection data with metals and the initial CT image. 


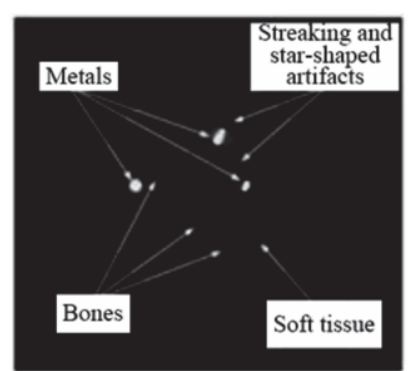

(a) Segmented metals

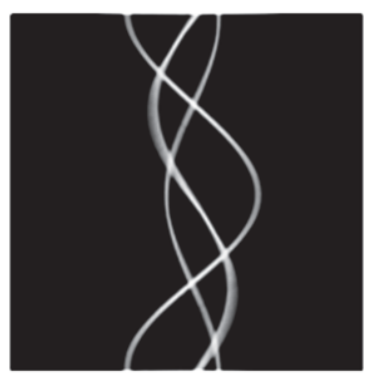

(b) Metal trace in projection

Fig. 3. The segmented metal and the trace in projection.

\subsection{Projection interpolation and reconstruction with no metal}

Now the projection data corresponding to the metal traces can be removed from the raw projection data and then interpolated using linear interpolation method and the projection data adjacent to metals at each angular position $\theta$. This operation can be expressed by the following Eq. (4). The CT image with no metal $\beta_{c}(x, y)$ are then reconstructed using $P_{c}(\theta, s)$ and the FBP algorithm in Eq. (1).

$$
P_{c}(\theta, s)=\left\{\begin{array}{lll}
P(\theta, s) & \text { if } & s_{m}(\theta)=0 \\
L I(P(\theta, s)) & \text { otherwise }
\end{array}\right.
$$

\subsection{The forward projection calculation}

The forward projection now can be produced by the re-projection method and expressed by

$$
\bar{P}(\theta, s)=\int_{l(x, y, s, \theta)} \beta_{c}(x, y) d l .
$$

Where $\bar{P}(\theta, s)$ is the forward projection and $l(x, y, s, \theta)$ represents the $\mathrm{x}$-ray path.

Some constraints may be applied to the image $\beta_{c}(x, y)$ before the forward projection calculation to improve the accuracy. For example, negative x-ray attenuation coefficient does not exist. So the gray values of pixels, which has a negative value in the image, can be set to be zero.

\subsection{Projection correction and reconstruction}

After the forward projection operation, a new set of corrected projection data $\bar{P}_{c}(\theta, s)$ is created by combining the raw experimental projection data $P(\theta, s)$ with the forward projection data $\bar{P}(\theta, s)$. The data corresponding to metals are replaced with the forward projection values. The data corresponding to other structures are kept at the raw value. This operation can be expressed by

$$
\bar{P}_{c}(\theta, s)= \begin{cases}P(\theta, s) & \text { if } \quad s_{m}(\theta)=0 \\ \bar{P}(\theta, s) & \text { otherwise. }\end{cases}
$$


Table 1

The physical properties of the objects contained in the sample

\begin{tabular}{llllll}
\hline Description & Soft Tissue & Bone & Titanium & Steel & Molybdenum \\
\hline Atomic Number(Z) & 7.4 & 12 & 22 & 26 & 42 \\
Density & 1.0 & 1.9 & 4.5 & 7.9 & 10.2 \\
Attenuation relative to water & 1 & 2.5 & 9 & 19 & 46 \\
Approximate CT value & 0 & 1500 & 8000 & 18000 & 45000
\end{tabular}

Using the updated projection data $\bar{P}_{c}(\theta, s)$, new estimate of the CT image with no metal artifacts can be generated by the FBP algorithm in Eq. (1).

The above six steps can be iterated for a given number typically from 1 to 20 or until convergence criteria are met. The final image is then obtained by adding together the corrected image and the segmented metals.

\section{Experiment}

In order to evaluate the validity of the proposed algorithm, an experiment was performed using a biomedical sample. CT scanning was executed using an X-ray CT scanner with 160KVA small focus $\mathrm{x}$-ray tube from Germany YXLON and a flat panel detector Paxscan 2520 from USA Varian.

The sample consists of soft tissue, bones and metals. In which, three steel needles with different crosssections were inserted into the soft tissue and the whole sample was fixed in a plastic container. The physical properties of the objects contained in the sample are shown in Table 1. It shows that the density of Steel is much higher than that of soft tissue and bones. The scanning parameters are as follows: sourceobject-distance $960 \mathrm{~mm}$, source-detector-distance $1210 \mathrm{~mm}$, detector channel size $0.254 \mathrm{~mm}$, step angle $0.5^{\circ}$ and the number of angular steps 720 . Figure 4 shows the pictures of the adopted CT scanning system and the sample.

Figure 2 shows the initial reconstruction image of the cross-section No.350, a typical slice, and its corresponding raw projection data. Clearly, the initial image is full of streak and star-shaped artifacts around the metals.

Figure 5 presents the CT images of some typical slices No. 350, 400, 450, 500, and 550 before and after correction. Figure 5 (a) shows the initial reconstructed images without correction. Figure 5 (b) shows the corrected image using the conventional linear interpolation method. Figure 5 (c) shows the corrected image using our proposed method. The adopted threshold value for metal segmentation is 0.7 (In our experiment, the pixel value is scaled to $\left[\begin{array}{ll}0 & 1\end{array}\right]$.). Four iterations are executed. By comparing Figure 5 (c)
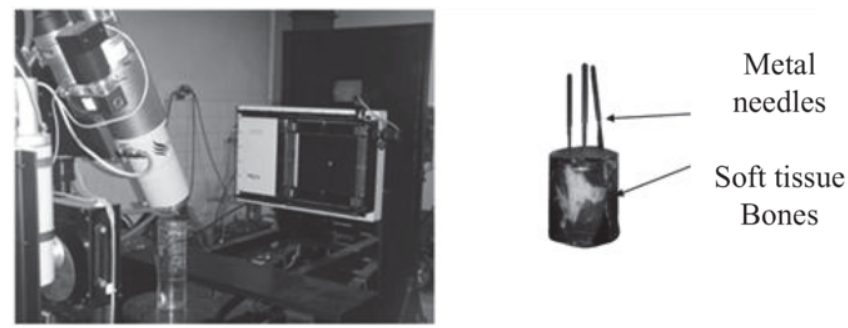

Fig. 4. The adopted CT scanning system and the sample. 


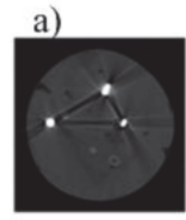

b) 350

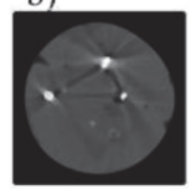

c)

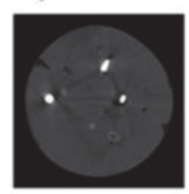

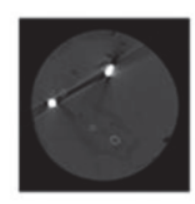

400
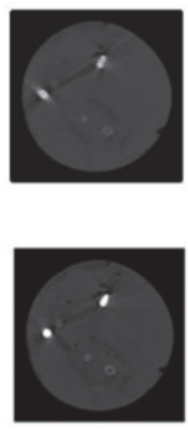

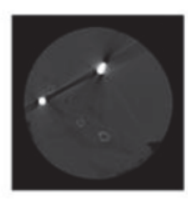

450
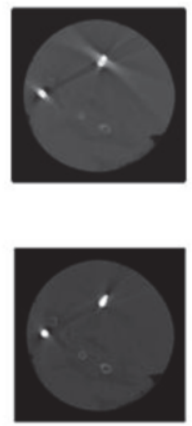

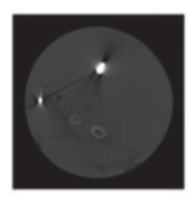

500
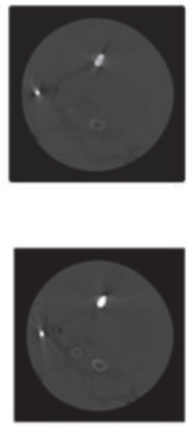

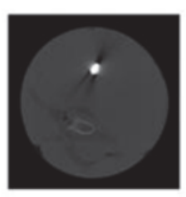

550
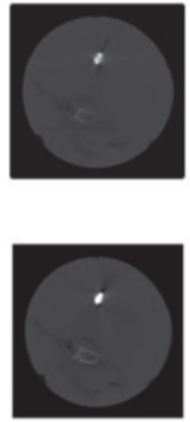

Fig. 5. The CT images of some typical slices before and after correction. (a) displays the initial reconstructed images without correction. (b) corresponds to the ones with the conventional linear interpolation correction. (c) is the result corrected by the proposed method.

with Figures 5 (a) and (b), it is evident that the image obtained using our proposed technique contains much fewer metal artifacts without image distortion, hence validating the presented method.

\section{Discussion and conclusion}

In this paper, a MAR algorithm is proposed and verified experimentally. Using our method, inaccurate metal artifact pixels in the original CT slices were removed while precise reconstruction of the soft-tissue was attained using forward projections with no metal information. Moreover, the proposed algorithm can effectively reduce metal artifacts without producing distortions in the non-metallic regions, thereby improving the diagnostic value of the image. Our work is of value to the medical and biological applications of X-ray CT. Of note, since our method requires manual adjustment of the segmentation threshold value and the reconstruction parameters, future work is needed to optimize the automatic determination of these parameters.

\section{Acknowledgement}

S. Nawaz thanks international school of Beijing university of aeronautics and astronautics for financial support to this work. This work was partly supported by the National Natural Science Foundation of China (grant no. 11179009), Program for New Century Excellent Talents in University (NCET) from Ministry of Education of P.R.China and Beijing NOVA program (2009A09).

\section{References}

[1] G. N. Hounsfield, Computerized transverse axial scanning (tomography): part 1 description of system, British Journal of Radiology 46 (1973), 1016-1022. 
[2] A. C. Kak and M. Slaney, Principles of Computerized Tomographic Imaging, IEEE press, New York, 1988, pp. 64-75.

[3] E. Klotz, W. A. Kalender and R. Sokiransky et al., Algorithms for the reduction of CT artefacts caused by metallic implants, SPIE 1234 (1991),642-650.

[4] G. Wang, S. L. Donald and J. A. O'Sullivan et al., Iterative deblurring for CT metal artifact reduction, IEEE Transactions on Medical Imaging 15 (1996), 657-664.

[5] B De Man, J. Nuyts, and P. Dupont et al., Metal streak artifact in X-ray computed tomography: a simulation study, IEEE Transactions on Nuclear Science 46 (1999), 691-696.

[6] S. Zhao, D. D. Robertson and G. Wang et al., X-ray CT metal artifact reduction using wavelets: an application for imaging total hip prostheses, IEEE Trans. Med. Imaging. 19 (2000), 1238-1247.

[7] B. De Man, J. Nuyts, and P. Dupont et al., Reduction of metal streak artifacts in x-ray computed tomography using a transmission maximum a posteriori algorithm, IEEE Transactions on Nuclear Science 47 (2000), 977-981.

[8] B. De Man, J. Nuyts, and P. Dupont et al., An iterative maximum-likelihood polychromatic algorithm for CT, IEEE Transactions on Medical Imaging 20 (2001), 699-1008.

[9] J. Wei, L. Chen and G. A. Sandison et al., X-ray CT high-density artifact suppression in the presence of bones, Phys. Med. Biol. 49 (2004), 5407-5418.

[10] J. F. Barrett and N. Keat, 3D computed tomography using a micro-focus X-ray source: Analysis of artifact formation in the reconstructed images using simulated as well as experimental projection data, Nuclear Instruments and Methods in Physics Research A 542 (2004), 1679-1691.

[11] S. Krimmela, J. Stephanb and J.Baumannb, Artifacts in CT: Recognition and avoidance, Radiographics 24 (2005),399407.

[12] F. P. Vidal, J. M. Letang, G. Peix and P. Cloetens, Investigation of artifact sources in synchrotron microtomography via virtual X-ray imaging, Nuclear Instruments and Methods in Physics Research B 234 (2005),333-348.

[13] K. Remeysen and R. Swennen, S. Krimmela, Beam hardening artifact reduction in microfocus computed tomography for improved quantitative coal characterization, International Journal of Coal Geology 67 (2006),101-111.

[14] H. Yu, K. Zeng, D. K. Bharkhada et al., A segmentation-based method for metal artifact reduction, Acad. Radio. 14 (2007), 495-504.

[15] J. Fu, H. N. Lu, L. Zhang and J. J. Sun, X-CT imaging method for large objects using double offset scan mode, Nuclear Instruments and Methods in Physics Research Section A: Accelerators, Spectrometers, Detectors and Associated Equipment 575 (2007),519-523.

[16] C. Lemmens, D. Faul and J. Nuyts, Suppression of metal artifacts in CT using a reconstruction procedure that combines MAP and projection completion, IEEE Transactions on Medical Imaging 28 (2009), 250-260.

[17] J. Fu, B. H. Jiang, B. Li, P. Li and Q. L. Wang, Methods determining the angular increment of a continuous scan cone-beam CT system, IEEE Transactions on Nuclear Science 57 (2010),1071-1076.

[18] J. Fu, P. Li, Q. L. Wang, S. Y. Wang, M. Bech, A. Tapfer, D. Hahn and F. Pfeiffer, A reconstruction method for equidistant fan beam differential phase contrast computed tomography, Phys. Med. Biol. 56, (2011), 4529-4538.

[19] J. Fu, A. Velroyen, R. B. Tan, J. W. Zhang, L. Y. Chen, A. Tapfer, M. Bech and F. Pfeiffer, A reconstruction method for cone-beam differential x-ray phase-contrast computed tomography, Optics Express 20, (2012), 21512-21519.

[20] J. Fu, S. Schleede, R. Tan, L. Chen, M. Bech, K. Achterhold, M. Gifford, R. Loewen, R. Ruth and F. Pfeiffer, An algebraic iterative reconstruction technique for differential x-ray phase-contrast computed tomography, Z. Med. Phys. 23, (2013), 186-193.

[21] J. Fu, Phase contrast computed tomography, in: Computed Tomography - Clinical Applications, L. Saba, ed., InTech, Croatia, 2012.

[22] M. Abdoli, R. A. Dierckx and H. Zaidi, Metal artifact reduction strategies for improved attenuation correction in hybrid PET/CT imaging, Medical Physics 39 (2012), 3343-3360.

[23] J. Fu and R. B. Tan, In-line phase contrast micro-CT reconstruction for biomedical specimens, Bio-Medical Materials and Engineering 24 (2014),431-437.

[24] J. Fu and L. Y. Chen, Single-slice reconstruction method for helical cone-beam differential phase-contrast CT, Bio-Medical Materials and Engineering 24 (2014),45-51.

[25] J. Fu, M. Willner, L. Chen, R. Tan, K. Achterhold, M. Bech, J. Herzen, D. Kunka, J. Mohr and F. Pfeiffer, Helical differential X-ray phase-contrast computed tomography, Phys. Med. 30 (2014),374-379.

[26] J. Fu, R. B. Tan and L. Y. Chen, Analysis and accurate reconstruction of incomplete data in X-ray differential phasecontrast computed tomography, Anal. Bioanal. Chem. 406 (2014),897-904. 